\title{
Chemical Constituents from Aegle marmelos
}

\section{Surat Laphookhieo,* Chalita Phungpanya, Cholpisut Tantapakul, Somsak Techa, Suphara Tha-in and Wanwasan Narmdorkmai}

\author{
Natural Products Research Laboratory, School of Science, Mae Fah Luang University, Tasud, \\ Muang, Chiang Rai 57100, Thailand
}

\begin{abstract}
Um novo produto natural derivado da oxazolina, chamado aeglemarmelosina (1), juntamente com oito compostos conhecidos (2-9), foi isolado a partir de raízes e ramos de Aegle marmelos. Os compostos 1-6 foram isolados das raízes e 7-9 dos galhos. Os compostos 5 e $\mathbf{6}$ também foram encontrados nos galhos. Todas as estruturas foram caracterizadas por métodos espectroscópicos de RMN uni e bidimensionais
\end{abstract}

A new natural product oxazoline derivative named aeglemarmelosine (1) along with eight known compounds (2-9) were isolated from roots and twigs of Aegle marmelos. Compounds 1-6 were isolated from the roots whereas compounds 7-9 were obtained from twigs. Compounds 5 and 6 were also detected from the twigs. All structures were characterized by extensive 1D and 2D NMR spectroscopic methods.

Keywords: Aegle marmelos, oxazoline, coumarins, alkaloids and Rutaceae

\section{Introduction}

Aegle marmelos, commonly known as "Ma Toom" in Thai, belongs to the Rutaceae family. Several parts of this plant have been used by the local Thai people in folk medicines. For example, the infusion of dried unripe fruits has been used as antidiarrhea and antidysentery agents, the juice from crushed leaves has been used for the treatment of bronchitis, and the decoction of root barks has also been used as anti-malarial drug. ${ }^{1}$ In addition, young leaves are used as vegetable. The chemical investigation of the leaves of this plant has revealed the presence of a number of alkaloids ${ }^{2-7}$ and coumarins. ${ }^{1,8,9}$ In our continuing study on chemical constituents from Thai medicinal plants, we now report herein the isolation and structure elucidation of a rare natural product oxazoline derivative product (1) along with eight known compounds (2-9) (Figure 1) from the roots and twigs of A. marmelos.

\section{Experimental}

\section{General procedure}

UV spectra were recorded with a Perkin-Elmer UVVis spectrophotometer. The IR spectra were recorded

*e-mail: surat@mfu.ac.th, suratlpk@gmail.com with a Perkin-Elmer FT-IR spectrophotometer. The ${ }^{1} \mathrm{H}$ and ${ }^{13} \mathrm{C}$ NMR spectra were recorded using $400 \mathrm{MHz}$ Bruker FT-NMR Ultra Shield spectrometer. Chemical shifts were recorded in parts per million $(\delta)$ in $\mathrm{CDCl}_{3}$<smiles>COc1ccc(C2CN=C(c3ccccc3)O2)cc1</smiles><smiles>O=c1ccc2cc3ccoc3c(O)c2o1</smiles><smiles>CC(C)=CCC(C)(C)C[Mg]</smiles>

$7: \mathrm{R}=\mathrm{Me}$<smiles>CC(C)(O)C1Cc2cc3ccc(=O)oc3cc2O1</smiles>

8<smiles>COc1ccc2c(OC)c3ccoc3nc2c1OC</smiles>

2<smiles>O=c1ccc2ccc(O)cc2o1</smiles>

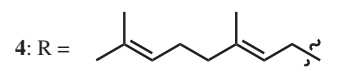<smiles>C/C(=C\C[Te])CCC1OC1C(C)C</smiles><smiles>C/C=C(/C)CC[C@@H](O)C(C)(C)O</smiles><smiles>COc1cc2ccc(=O)oc2cc1O</smiles>

Figure 1. Compounds isolated from A. marmelos. 
with tetramethylsilane (TMS) as an internal reference. High resolution mass spectra were obtained using Bruker microTOF mass spectrometer. Dried flash column chromatography and column chromatography (CC) were carried out on silica gel $60 \mathrm{H}$ (Merck, 5-40 $\mu \mathrm{m}$ ) and silica gel 100 (Merck, 63-200 $\mu \mathrm{m}$ ), respectively. Precoated plates of silica gel $60 \mathrm{~F}_{254}$ were used for analytical purposes.

\section{Plant material}

The roots and twigs of A. marmelos were collected from Chiang Rai Province, northern part of Thailand, in December 2008. Botanical identification was achieved through comparison with a voucher specimen number QBG 33029 in the Herbarium Collection of Queen Sirikit Garden, Mae Rim District, Chiang Mai, Thailand.

\section{Extraction and isolation}

The roots $(160 \mathrm{~g})$ and the twigs $(4.5 \mathrm{~kg})$ of A. marmelos were extracted with dichloromethane $\left(\mathrm{CH}_{2} \mathrm{Cl}_{2}\right)$, over a period of 3 days, at room temperature, and evaporated under reduced pressure, to provide crude $\mathrm{CH}_{2} \mathrm{Cl}_{2}$ extracts of roots $(8.40 \mathrm{~g})$ and twigs $(15.34 \mathrm{~g})$.

The $\mathrm{CH}_{2} \mathrm{Cl}_{2}$ extract from the roots was chromatographed by dried flash column chromatography over silica gel and eluted with a gradient of hexane-EtOAc (10:0, $9: 1,8: 2,7: 3,6: 4,5: 5,4: 6,3: 7,2: 8,1: 9,0: 10, \mathrm{v} / \mathrm{v})$, to afford compound $2(504 \mathrm{mg})$ and twenty-four fractions (MTC_1A-MTC_1Z). Fraction MTC_1D (48 mg) was purified by preparative TLC with acetone-hexane $(1: 5, \mathrm{v} / \mathrm{v})$ to afford compound $\mathbf{4}(10 \mathrm{mg})$. Compound $\mathbf{5}$ $(10 \mathrm{mg})$ was derived from Fraction MTC_1G (40 mg) by preparative TLC with $100 \% \mathrm{CHCl}_{3}$. Fraction MTC_1J (36 mg) was further purified by preparative TLC with $\mathrm{CH}_{2} \mathrm{Cl}_{2}$-hexane $(4: 1, \mathrm{v} / \mathrm{v})$ to give compounds $\mathbf{1}(9.7 \mathrm{mg})$ and $3(5.7 \mathrm{mg})$. Fractions MTC_1L (1.4 g) was subjected to $\mathrm{CC}\left(\mathrm{CH}_{2} \mathrm{Cl}_{2}\right.$-hexane, $\left.1: 1, \mathrm{v} / \mathrm{v}\right)$ to give compounds 3 $(50 \mathrm{mg})$ and $\mathbf{6}(165 \mathrm{mg})$.

The $\mathrm{CH}_{2} \mathrm{Cl}_{2}$ extract from the twigs was subjected to dried flash column chromatography over silica gel and eluted with a gradient of hexane-EtOAc (10:0, 9:1, 8:2, $7: 3,6: 4,5: 5,4: 6,3: 7,2: 8,1: 9,0: 10, \mathrm{v} / \mathrm{v})$, to afford ten fractions. Fraction AMD3 $(631.5 \mathrm{mg}$ ) was repeated dried flash column chromatography with $100 \% \mathrm{CH}_{2} \mathrm{Cl}_{2}$, to give compounds $8(25.2 \mathrm{mg})$ and $\mathbf{9}(25.6 \mathrm{mg})$. Compounds $\mathbf{5}$ $(5.97 \mathrm{mg})$ and $7(8.4 \mathrm{mg})$ were derived from fraction AMD5 $(1.11 \mathrm{~g})$ by repeated dried flash column chromatography with $\mathrm{CH}_{2} \mathrm{Cl}_{2}$-hexane $(3: 7, \mathrm{v} / \mathrm{v})$. Fraction AMD6 $(1.37 \mathrm{~g})$ was further purified by $\mathrm{CC}$ with $\mathrm{MeOH}-\mathrm{CH}_{2} \mathrm{Cl}_{2}(1: 99, \mathrm{v} / \mathrm{v})$, to yield compound $\mathbf{6}$ (79.5 mg).

\section{Aeglemarmelosine (1)}

Orange viscous oil; $[\alpha]_{\mathrm{D}}^{27}+7.89^{\circ}\left(c 0.20, \mathrm{CHCl}_{3}\right) ; \mathrm{UV}$ $\left(\mathrm{CHCl}_{3}\right) \lambda_{\text {max }} / \mathrm{nm}$ : 240; IR (neat) $v_{\text {max }} / \mathrm{cm}^{-1}: 2918,2489$, 1650, 1615, 1515, 1248, 756; ${ }^{1} \mathrm{H}$ NMR (400 MHz, $\left.\mathrm{CDCl}_{3}\right)$ : $\delta 8.10\left(2 \mathrm{H}, \mathrm{m}, \mathrm{H}-2^{\prime} / \mathrm{H}-6^{\prime}\right), 7.48\left(1 \mathrm{H}, \mathrm{tt}, J 8.0,2.0 \mathrm{~Hz}, \mathrm{H}-4^{\prime}\right)$, $7.42\left(2 \mathrm{H}, \mathrm{m}, \mathrm{H}-3^{\prime} / \mathrm{H}-5^{\prime}\right), 7.28$ (2H, d, J $\left.8.8 \mathrm{~Hz}, \mathrm{H}-2^{\prime \prime} / 6^{\prime \prime}\right)$, $6.91\left(2 \mathrm{H}, \mathrm{d}, J 8.8 \mathrm{~Hz}, 3^{\prime \prime} / 5^{\prime \prime}\right), 5.51(1 \mathrm{H}, \mathrm{dd}, J 10.0,8.0 \mathrm{~Hz}$, H-5), 4.44 (1H, dd, J 14.8, $10.0 \mathrm{~Hz}, \mathrm{H}-4 \mathrm{a}), 3.99$ (1H, dd, $J 14.8,8.0 \mathrm{~Hz}, 4 \mathrm{~b})$, and 3.81 (3H, s, $4 "-\mathrm{OMe}) ;{ }^{13} \mathrm{C} \mathrm{NMR}$ $\left(100 \mathrm{MHz}, \mathrm{CDCl}_{3}\right): \delta 164.0(\mathrm{C}-2), 159.7$ (C-4"), 132.9 (C-1") $131.4\left(\mathrm{C}-4^{\prime}\right), 128.6$ (C-1'), $128.4\left(\mathrm{C}-2^{\prime}\right.$ and $\left.\mathrm{C}-6^{\prime}\right)$, 128.3 (C-3' and C-5'), 127.4 (C-2" and C-6"), 114.2 (C$3^{\prime \prime}$ and $\left.\mathrm{C}-5^{\prime \prime}\right), 81.1$ (C-5), 62.9 (C-4) and $55.3\left(4^{\prime \prime}-\mathrm{OMe}\right)$; HR-MS (APCI, +ve) $\mathrm{m} / z 254.1180[\mathrm{M}+\mathrm{H}]^{+}$(calc. for $\left.\mathrm{C}_{16} \mathrm{H}_{16} \mathrm{NO}_{2}, 254.1181\right)$.

\section{Antimalarial assay}

Antimalarial activity was evaluated against the parasite Plasmodium falciparum ( $\mathrm{K}_{1}$, mutidrug resistant), using the method of Trager and Jensen. ${ }^{10}$ Quantitative assessment of in vitro malarial activity was determined by means of the microculture radioisotope technique based on the method described by Desjardins et al. ${ }^{11}$ The inhibitory concentration $\left(\mathrm{IC}_{50}\right.$ ) represented the concentration that caused $50 \%$ reduction in parasite growth which was indicated by the in vitro uptake of $\left[{ }^{3} \mathrm{H}\right]$-hypoxanthine by $P$. falciparum. The standard compound was dihydroartemisinin $\left(\mathrm{IC}_{50} 4.1 \mathrm{nmol} \mathrm{L}^{-1}\right)$.

\section{Results and Discussion}

Aeglemarmelosine $(\mathbf{1})[\alpha]_{\mathrm{D}}^{27}+7.89^{\circ}\left(c 0.20, \mathrm{CHCl}_{3}\right)$ was isolated as an orange viscous oil. A molecular formula, $\mathrm{C}_{16} \mathrm{H}_{15} \mathrm{NO}_{2}$, was established by HR-MS analysis of its ion peak $[\mathrm{M}+\mathrm{H}]^{+}$at $\mathrm{m} / \mathrm{z} 254.1180$ (calc. for $\mathrm{C}_{16} \mathrm{H}_{16} \mathrm{NO}_{2} \mathrm{~m} / \mathrm{z}$ 254.1181). The ${ }^{1} \mathrm{H}$ NMR spectral data of $\mathbf{1}$ (Table 1 ) showed the characteristic of oxazoline framework ${ }^{2}$ at $\delta 5.51(\mathrm{dd}$, 10.0, $8.0 \mathrm{~Hz}), 4.44(\mathrm{dd}, 14.8,10.0 \mathrm{~Hz})$ and $3.99(\mathrm{dd}, 14.8$, $8.0 \mathrm{~Hz}$ ) which were identified for $\mathrm{H}-5$, and $\mathrm{H}-4 \mathrm{a}$ and $\mathrm{H}-4 \mathrm{~b}$, respectively. The remaining ${ }^{1} \mathrm{H}$ NMR spectral data could be characterized as being due to a monosubstituted aromatic ring $\left[\delta 8.10\left(\mathrm{~m}, 2 \mathrm{H}, \mathrm{H}-2^{\prime}\right.\right.$ and $\left.\mathrm{H}-6^{\prime}\right), 7.42\left(\mathrm{~m}, \mathrm{H}-3^{\prime}\right.$ and $\left.\mathrm{H}-5^{\prime}\right)$ and 7.48 (tt, 8.0, $\left.2.0 \mathrm{~Hz}, \mathrm{H}-4^{\prime}\right)$ ], and a 4-methoxyphenyl group (1,4-disubstituted aromatic ring) [ $\delta 7.28(\mathrm{~d}, 8.8 \mathrm{~Hz}$, $2 \mathrm{H}, \mathrm{H}-2^{\prime \prime}$ and $\left.\mathrm{H}-6^{\prime \prime}\right), 6.91$ (d, 8.8 Hz, 2H, H-3" and H-5") and 3.81 (s, 4"-OMe)]. The monosubstituted aromatic ring was connected to $\mathrm{C}-2$ of the oxazoline ring because of the ${ }^{2} J$ and ${ }^{3} J$ connectivity of $\mathrm{H}-2^{\prime}(\delta 8.10)$ and $\mathrm{H}-3^{\prime}(\delta$ $7.42)$, respectively, to $\mathrm{C}-2(\delta$ 164.0) in HMBC correlation 
(Figure 2), whereas the 4-methoxyphenyl group was placed on $\mathrm{C}-5$ of the oxazoline ring because the H-5 $(\delta 5.51)$ and $\mathrm{H}-4(\delta 4.44$ and 3.99) showed HMBC correlation to $\mathrm{C}-1$ " $(\delta$ 132.9). The oxazoline derivative $\mathbf{1}$, therefore, was identified as being aeglemarmelosine (2-phenyl-5-(4methoxyphenyl)- $\Delta^{2}$-oxazoline) which has been reported as a synthetic compound by Callens et al. ${ }^{12}$

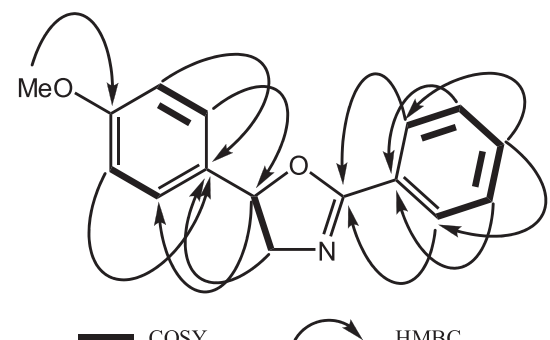

Figure 2. COSY and key HMBC correlations of aeglemarmelosine (1).

The remaining compounds were characterized as skimmianine (2), ${ }^{13}$ imperatorin (3), ${ }^{14}$ aurapten (4), ${ }^{15}$ epoxyaurapten (5), ${ }^{15}$ marmin $(\mathbf{6}),{ }^{15}$ xanthotoxin (7), ${ }^{14}$ marmisin $(\mathbf{8})^{16}$ and scopoletin $(\mathbf{9})^{17}$ by using extensive 1D and 2D NMR spectroscopy and by comparing of their spectral data with reported values. All compounds were tested for their antimalarial activity but, unfortunately, they were inactive.

\section{Supplementary Information}

Supplementary data are available free of charge at http://jbcs.sbq.org.br, as PDF file.

\section{Acknowledgments}

This work was supported by the Thailand Research Fund (grant number RSA5280011) and Mae Fah Luang University. We are indebted to Mr. Nitirat Chimnoi, Chulabhorn Research Institute, Bangkok, for recoding the mass spectra, and Assoc. Prof. Dr. Uma Prawat and Ms. Nareerat Thongtip, Department of Chemistry, Rajabhat Phuket University, Phuket, for recording the NMR spectra.

\section{References}

1. Mishra, B. B.; Singh, D. D.; Kishore, N.; Tiwari, V. K.; Tripathi, V. Phytochemistry 2010, 71, 230.

2. Phuwapraisirisan, P.; Puksasook, T.; Jong-aramruang, J.; Kokpol, U.; Bioorg. Med. Chem. Lett. 2008, 18, 4956.

3. Faizi, S.; Farooqi, F.; Zikr-Ur-Rehman, S.; Naz, A.; Noor, F.; Ansari, F.; Ahmad A.; Khan, S. A.; Tetrahedron 2009, 65, 998.

4. Manandhar, M. D.; Shoeb, A.; Kapil, R. S.; Popli, S. P.; Phytochemistry 1978, 17, 1814.

5. Basu, D.; Sen, R.; Phytochemistry 1974, 13, 2329.

6. Sharma, B. R.; Rattan, R. K.; Sharma, P.; Phytochemistry 1981, 20, 2606.

7. Govindachari, T. R.; Premila, M.S.; Phytochemistry 1983, 22, 755.

8. Ali, M. S.; Pervez, M. K.; Nat. Prod. Res. 2004, 18, 141.

9. Ohashi, K.; Watanabe, H.; Ohi, K.; Arimoto, H.; Okumura, Y.; Chem. Lett. 1995, 24, 881.

10. Trager, W.; Jensen, J. B.; Science 1976, 193, 673.

11. Desjardins, R. E.; Canfield, C. J.; Haynes, J. D.; Chulay, J. D.; Antimicrob. Agents Chemother. 1979, 16, 710.

12. Callens, R.; Anteunis, M. J. O.; De Witte, M.; Bull. Soc. Chim. Belg. 1987, 96, 619.

13. Inada, A.; Ogasawara, R.; Koga, I.; Nakatami, N.; Inatomi, Y.; Murata, H.; Nishi, M.; Naganishi, T.; Chem. Pharm. Bull. 2008, $56,727$.

14. Masuda, T.; Takasugi, M.; Anetai, M.; Phytochemistry 1998, 47, 13.

15. Chen, I. S.; Lin, Y. C.; Tsai, I. L.; Teng, C. M.; Ko, F. N.; Ishikawa, T.; Ishii, H.; Phytochemistry 1995, 39, 1091.

16. Rondest, J.; Das, B. C.; Ricroch, M. N.; Kan-Fan, C.; Potier, P.; Polonsky, J.; Phytochemistry 1968, 7, 1019.

17. Cassady, J. M.; Ojima, N.; Chang, C. J.; McLaughlin, J. L.; J. Nat. Prod. 1979, 42, 274.

Submitted: January 4, 2010 Published online: August 10, 2010 


\section{Supplementary Information}

\section{Chemical Constituents from Aegle marmelos}

Surat Laphookhieo,* Chalita Phungpanya, Cholpisut Tantapakul, Somsak Techa, Suphara Tha-in and Wanwasan Narmdorkmai

Natural Products Research Laboratory, School of Science, Mae Fah Luang University, Tasud, Muang, Chiang Rai 57100, Thailand<smiles>O=[N+]([O-])Oc1ccc(C2CN=C(c3ccccc3)O2)cc1</smiles>

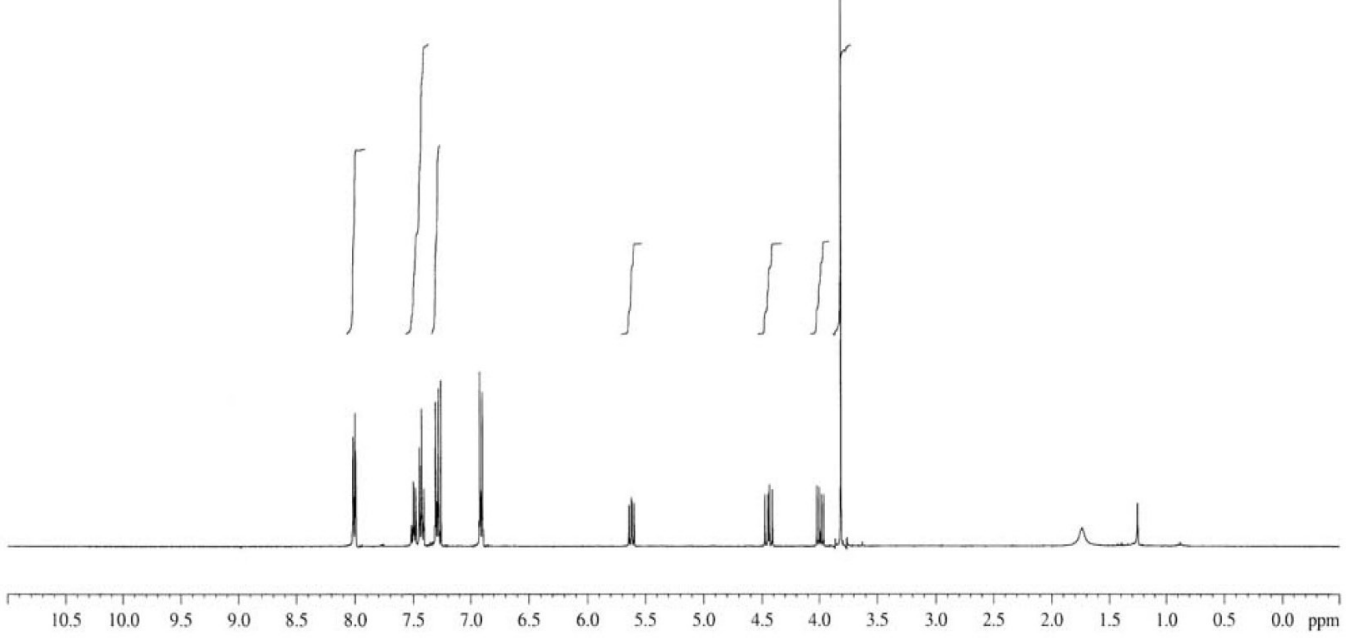

Figure S1. ${ }^{1} \mathrm{H}$ NMR spectrum of $\mathbf{1}\left(400 \mathrm{MHz}, \mathrm{CDCl}_{3}\right)$. 


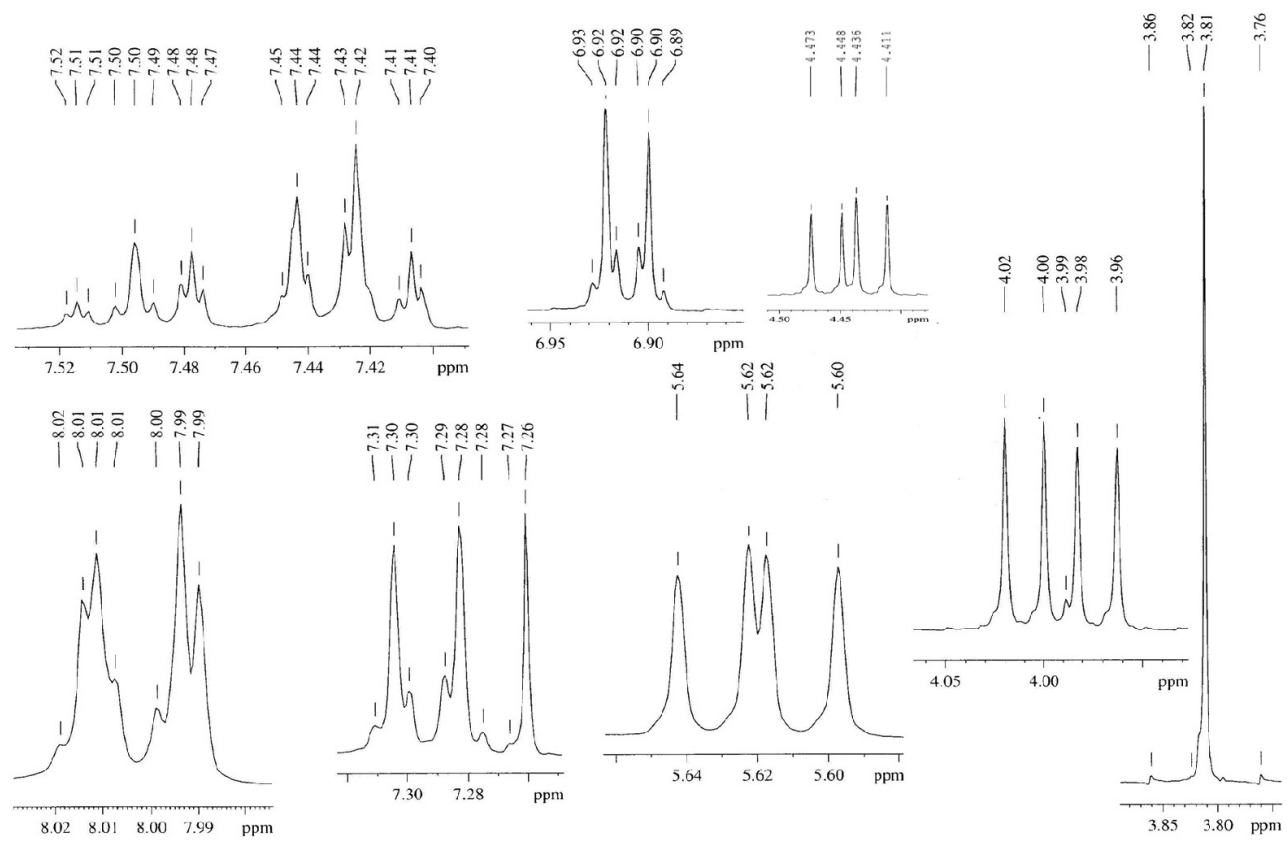

Figure S2. ${ }^{1} \mathrm{H}$ NMR spectrum of $\mathbf{1}$ (expanded) $\left(400 \mathrm{MHz}, \mathrm{CDCl}_{3}\right)$.

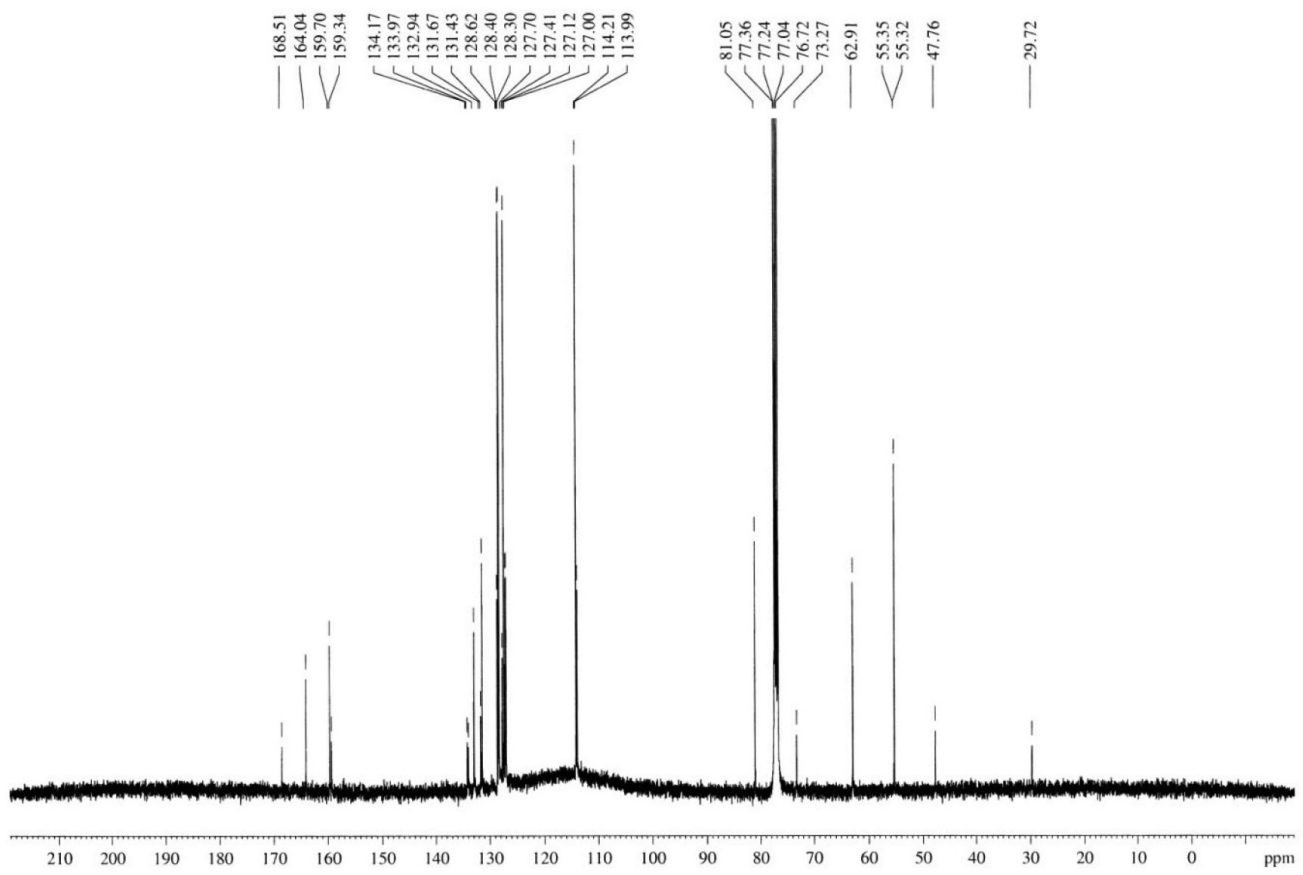

Figure S3. ${ }^{13} \mathrm{C}$ NMR spectrum of $\mathbf{1}\left(100 \mathrm{MHz}, \mathrm{CDCl}_{3}\right)$. 


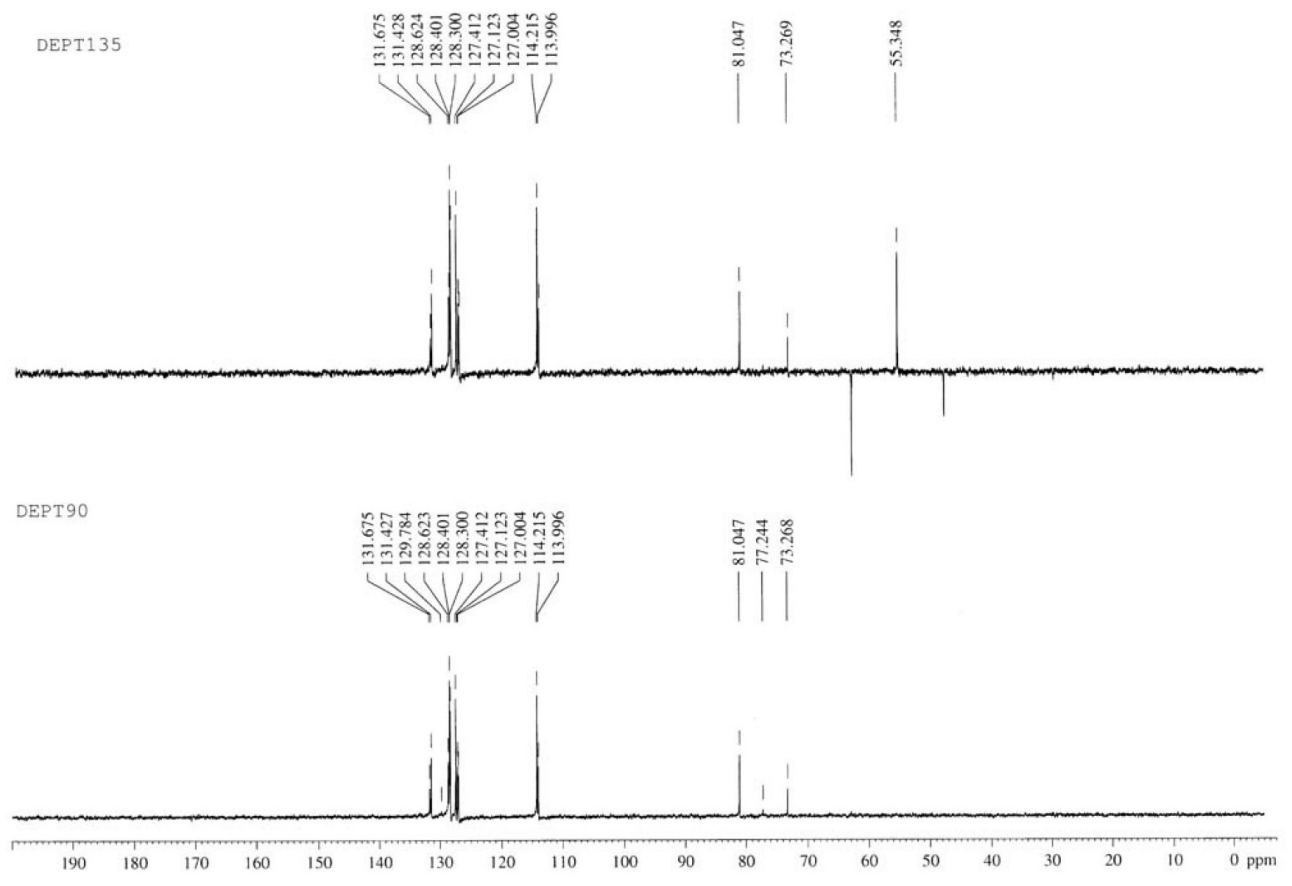

Figure S4. DEPT 135 and 90 spectra of $\mathbf{1 .}$

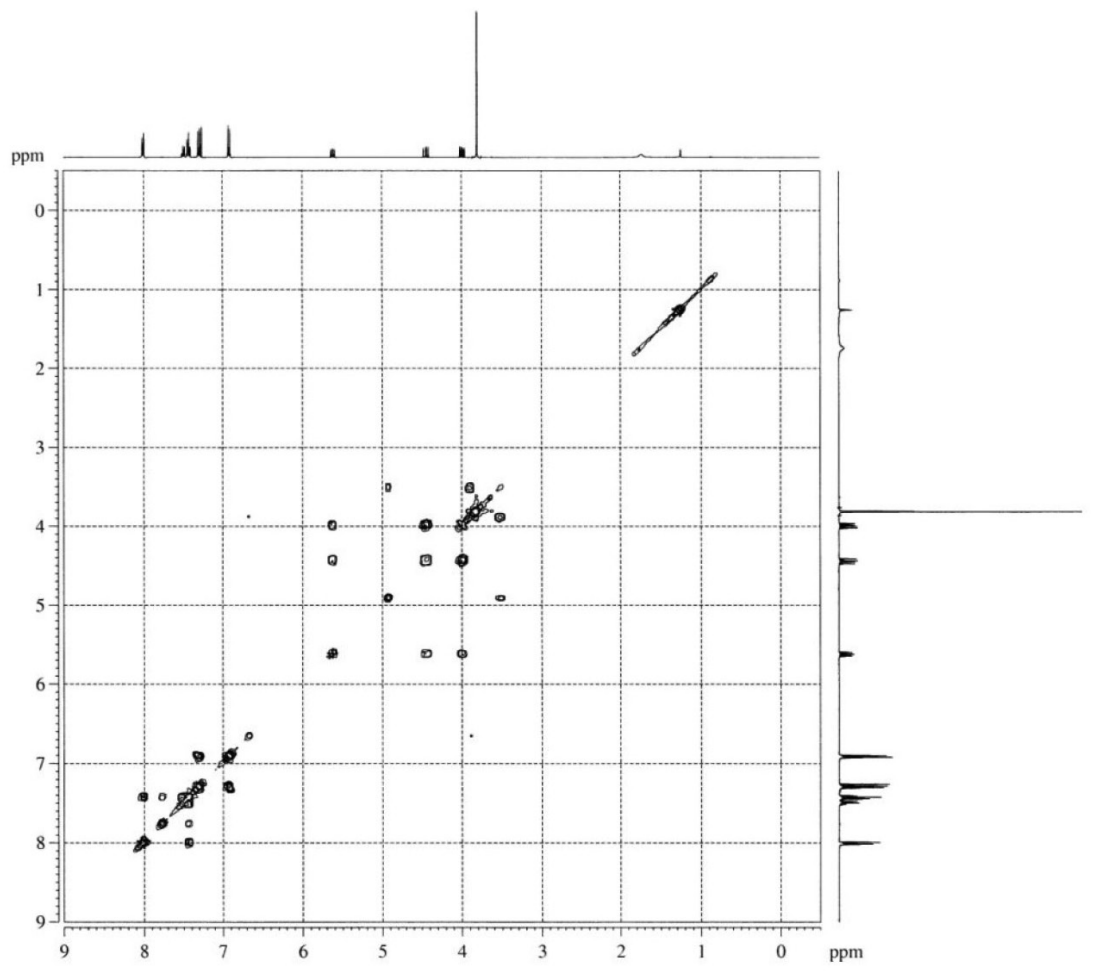

Figure S5. COSY spectrum of $\mathbf{1}$. 


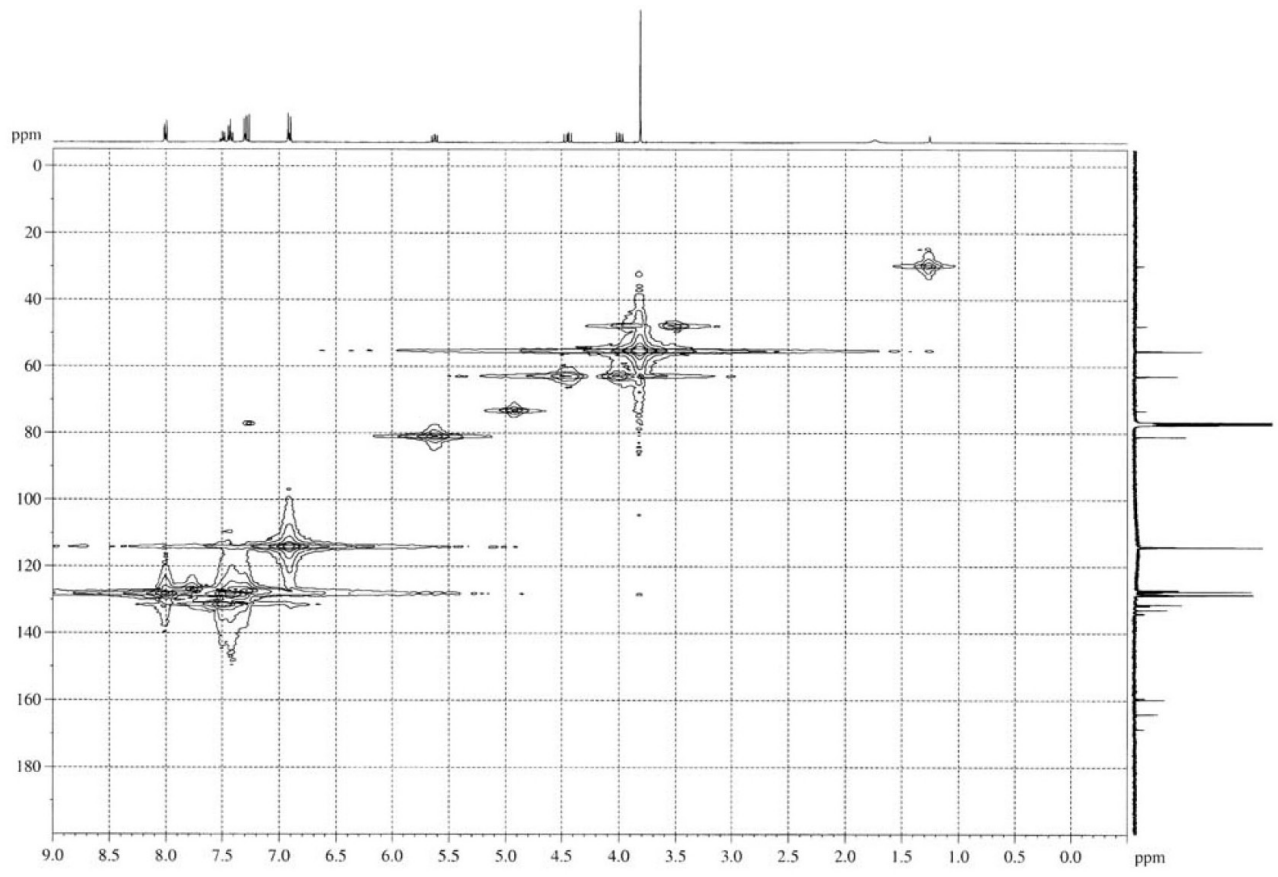

Figure S6. HMQC spectrum of $\mathbf{1}$.

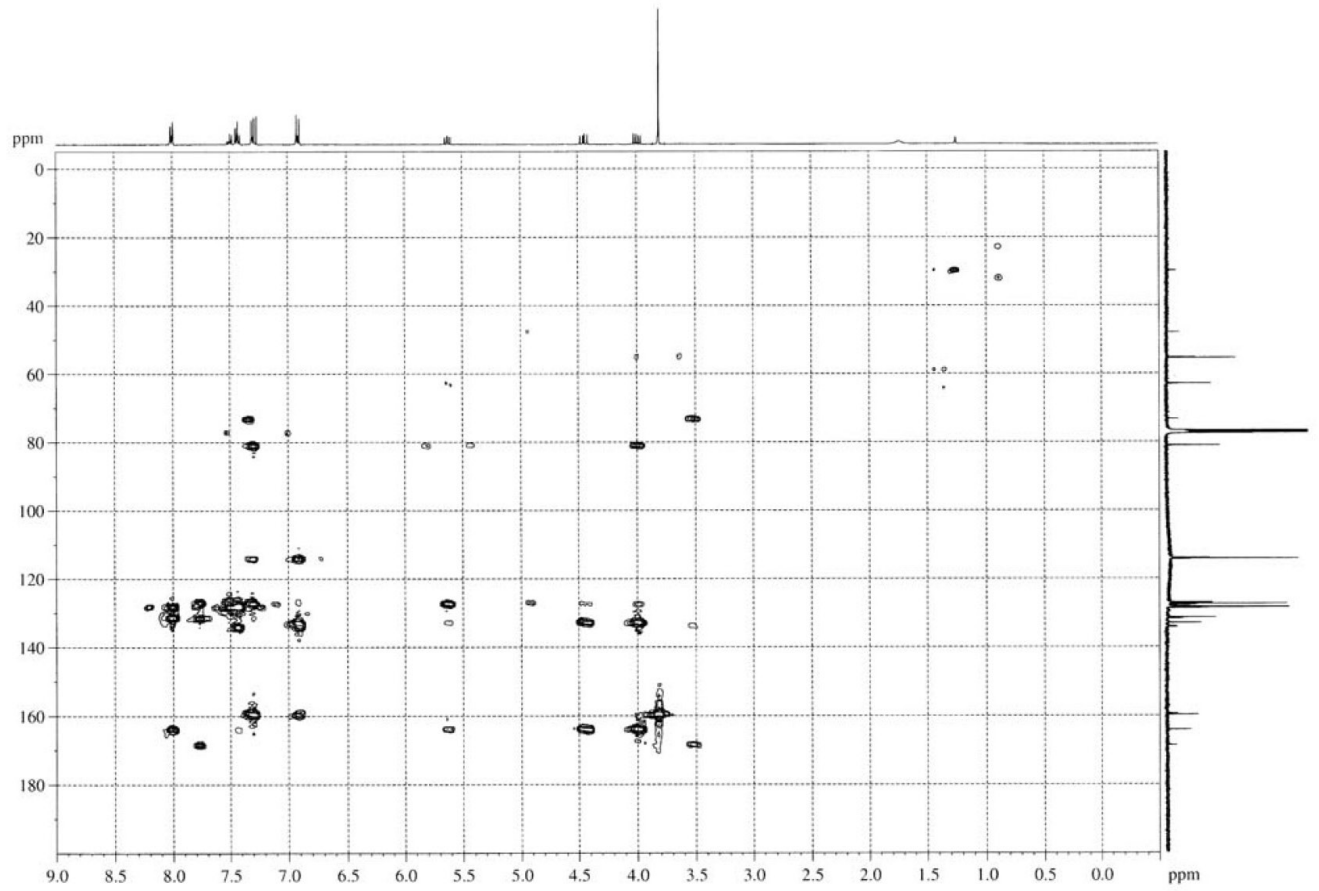

Figure S7. HMBC spectrum of $\mathbf{1}$. 


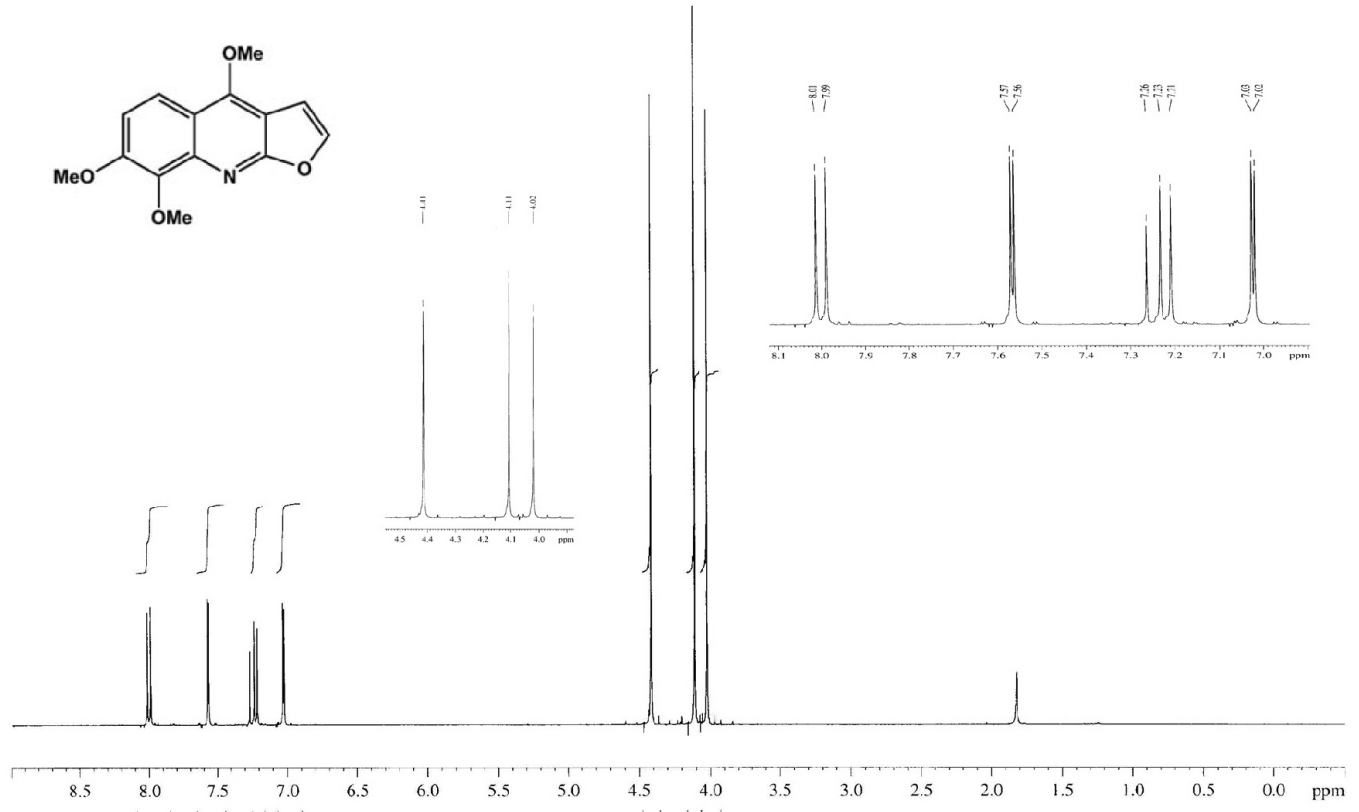

Figure S8. ${ }^{1} \mathrm{H}$ NMR spectrum of $2\left(400 \mathrm{MHz}, \mathrm{CDCl}_{3}\right)$.

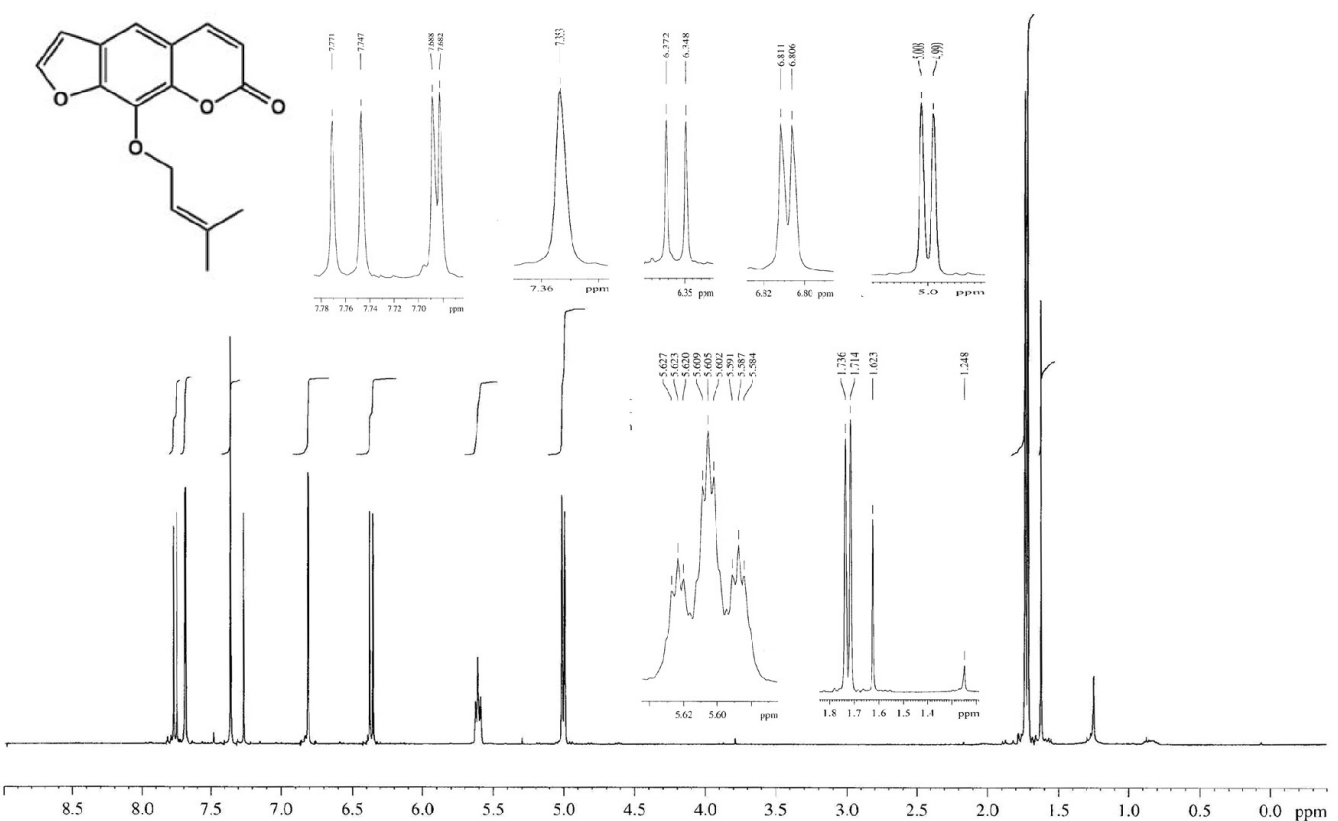

Figure S9. ${ }^{1} \mathrm{H}$ NMR spectrum of $\mathbf{3}\left(400 \mathrm{MHz}, \mathrm{CDCl}_{3}\right)$. 


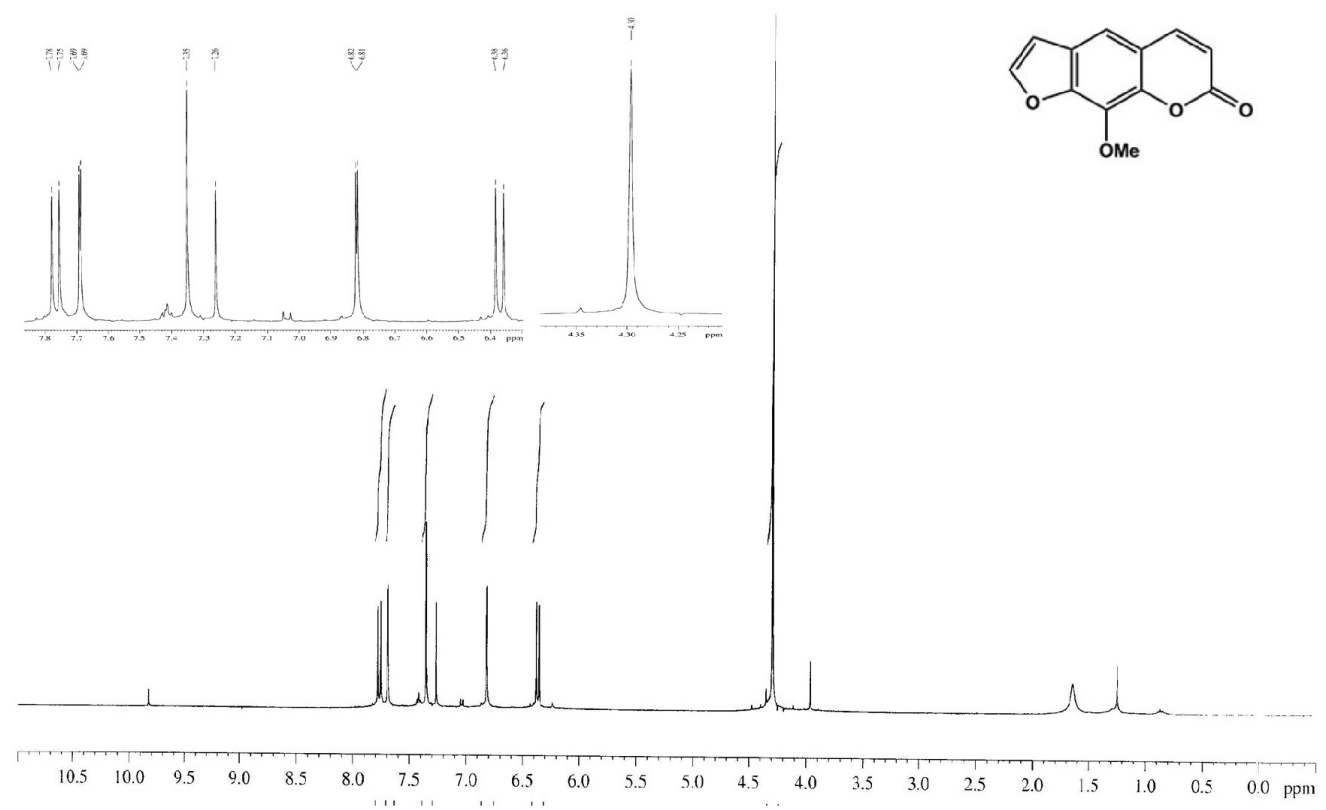

Figure S10. ${ }^{1} \mathrm{H}$ NMR spectrum of $4\left(400 \mathrm{MHz}, \mathrm{CDCl}_{3}\right)$.

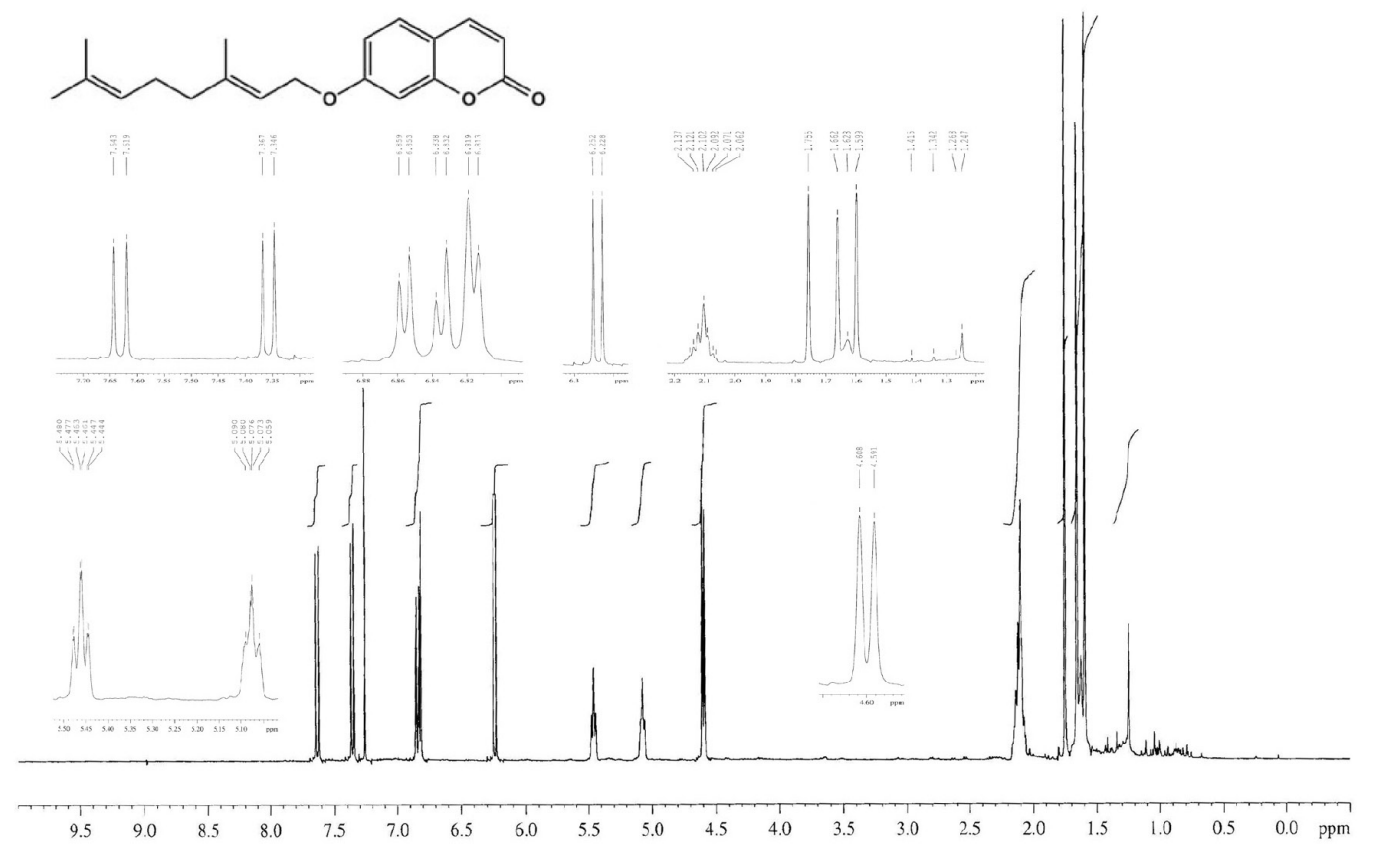

Figure S11. ${ }^{1} \mathrm{H}$ NMR spectrum of $5\left(400 \mathrm{MHz}, \mathrm{CDCl}_{3}\right)$. 
Vol. 22, No. 1, 2011

Laphookhieo et al.

ST
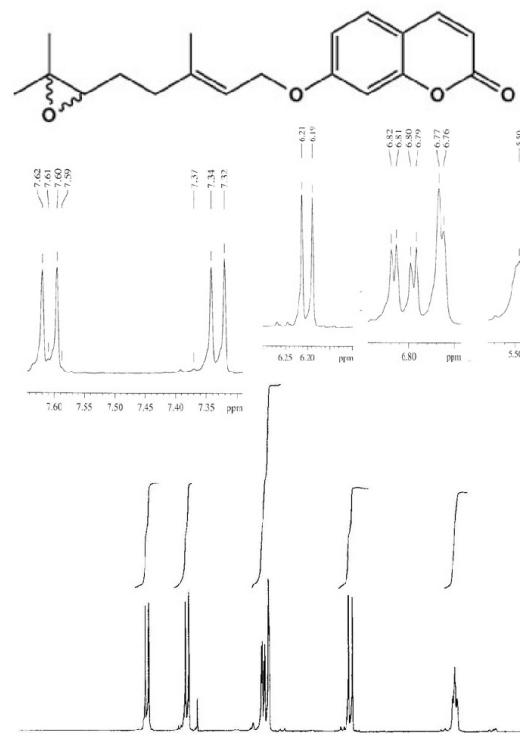

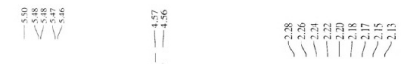

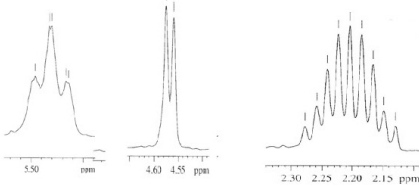

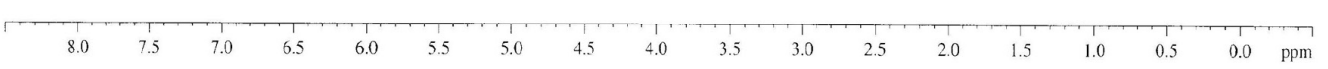

Figure S12. ${ }^{1} \mathrm{H}$ NMR spectrum of $6\left(400 \mathrm{MHz}, \mathrm{CDCl}_{3}\right)$.
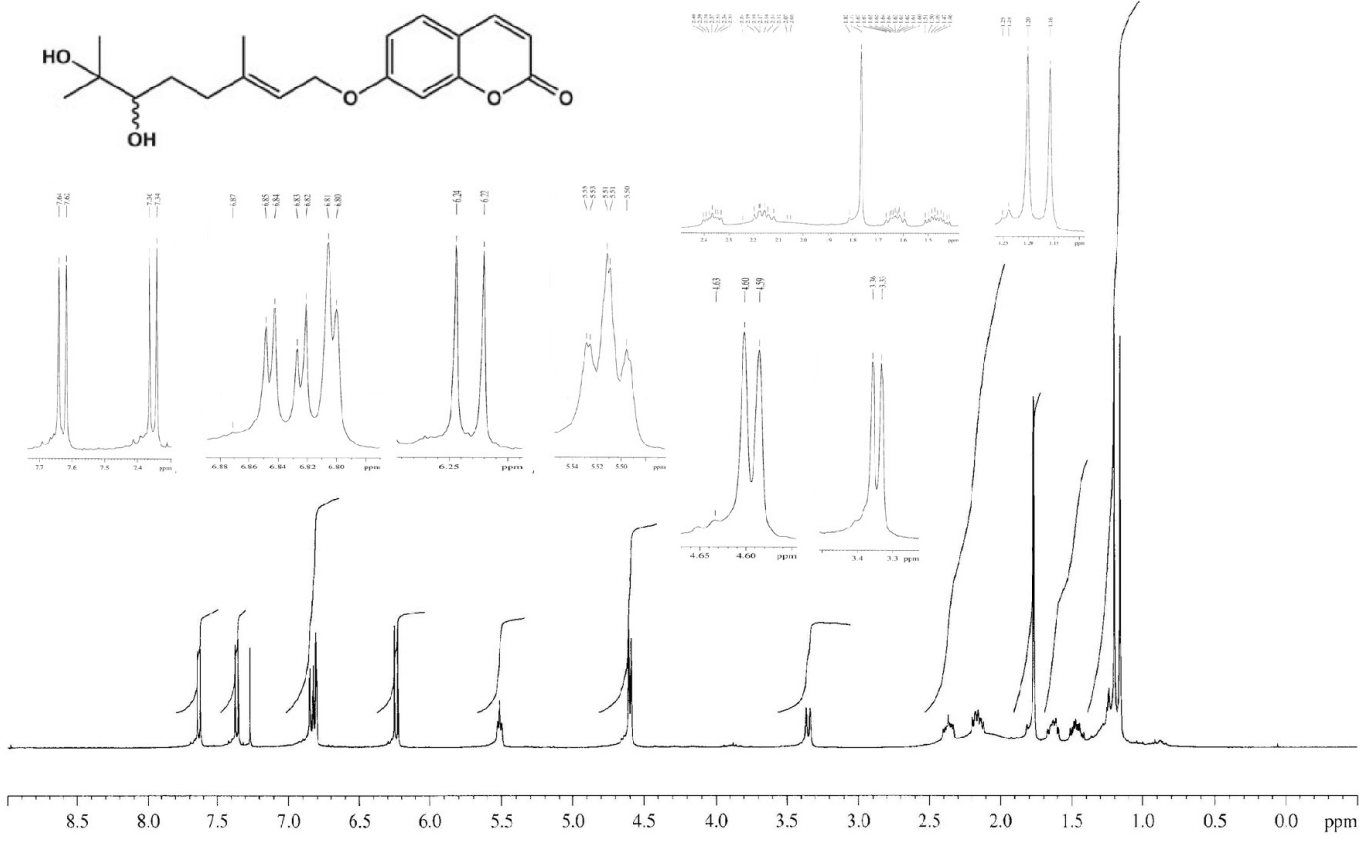

Figure S13. ${ }^{1} \mathrm{H}$ NMR spectrum of $7\left(400 \mathrm{MHz}, \mathrm{CDCl}_{3}\right)$. 
SB

Chemical Constituents from Angle marmelos

J. Braz. Chem. Soc.
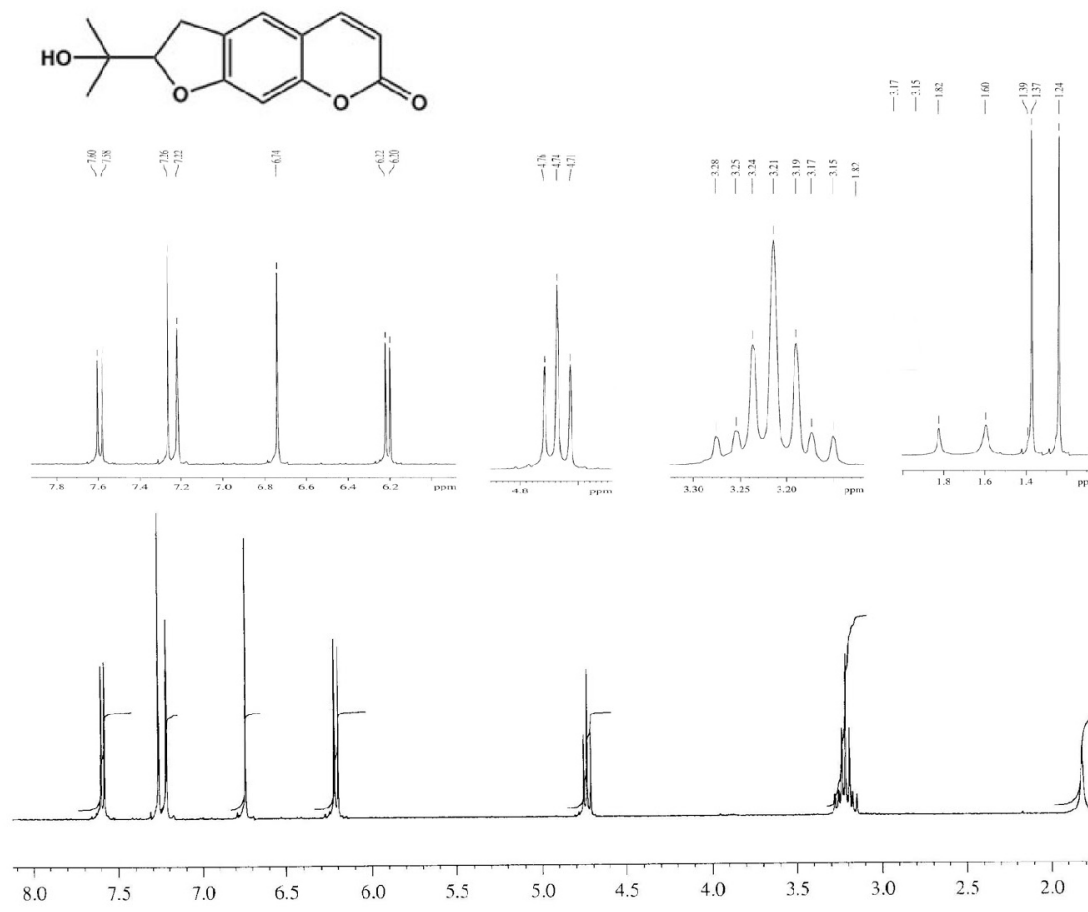
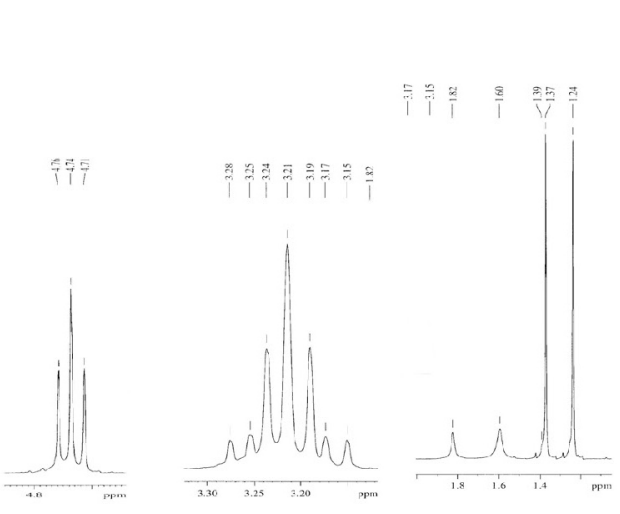

铰事

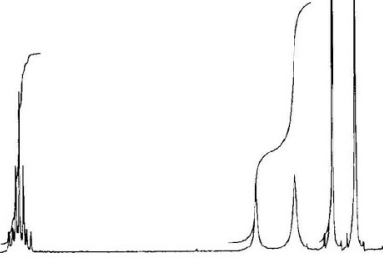

